\title{
Topological valley plasmon transport in graphene bi-layer metasurfaces: applications to sensing nanodevices
}

\author{
Yupei Wang, Jian Wei You, Zhihao Lan, and Nicolae C. Panoiu \\ Department of Electronic and Electrical Engineering, University College London, Torrington \\ Place, London WC1E 7JE, United Kingdom
}

\begin{abstract}
Topologically protected plasmonic states with wide topological band gaps provide unprecedented robustness against disorder-induced backscattering. In this study, we design a graphene bi-layer metasurface that possesses valley-Hall topological plasmonic modes in a nontrivial bandgap. In particular, the breaking of spatial-inversion symmetry of two graphene layers is achieved via a horizontal shift of the hole lattice of the top layer, which leads to topologically protected edge modes in the nontrivial bandgap. The corresponding band dispersion of the topological edge modes shows unidirectional propagation features. Moreover, we have designed a sensitive molecular sensor based on such graphene bi-layer metasurfaces, using the fact that the chemical potential of graphene varies upon adsorption of gas molecules. This effect leads to a marked variation of the transmission of the topological mode, and thus a sensing device with large sensitivity can be realized.
\end{abstract}

Keywords: Topological photonics, valley-Hall, graphene, sensing

\section{INTRODUCTION}

Topologically protected states are promising edge states inspired by the quantum Hall effect of topological insulators. ${ }^{1-3}$ Topological modes have novel and unique features, such as unidirectional propagation, are robust against imperfections, and do not display disorder-induced backscattering, properties that have found use in new integrated photonic devices. ${ }^{2-4}$ Topological edge modes could be achieved by breaking the symmetry-protected feature responsible for the generation of Dirac cones. For example, the time-inversion symmetry could be broken by adding an external magnetic field whereas the spatial-inversion symmetry can be broken by introducing an asymmetric perturbation in a structure with inversion symmetry. Importantly, many photonic platforms, such as meta-waveguides, photonic crystals, and metamaterials, are suitable for the implementation of the new ideas emerging from topological photonics. ${ }^{3-5}$

Degrees of freedom commonly used in condensed matter physics, such as spin and valley, have recently been introduced in the topological photonics. ${ }^{1-4,6}$ Two-dimensional (2D) materials with honeycomb lattice, such as graphene, exhibit nontrivial Berry curvature, and therefore can be used to study quantum phenomena such as the valley-Hall effect. ${ }^{3,4,6,7}$ By breaking the spatial-inversion symmetry in such materials, two photonic edge modes with opposite propagation directions could be achieved, their propagation direction being determined by the sign of the valley Chern number. ${ }^{7,8}$

The valley-Hall topological photonic modes of $2 \mathrm{D}$ materials have not been widely studied yet. ${ }^{9}$ In order to alleviate this problem, in this paper we design a bi-layer graphene metasurface to realize valley topological plasmonic modes by utilizing a novel mechanism of mirror symmetry breaking between the top and bottom graphene layers, by horizontally shifting in opposite directions the lattice of holes of the top layer. Furthermore, topologically guided valley modes are observed along a domain-wall interface constructed by placing two metasurfaces together in a mirror symmetric manner. Employing this feature of unidirectional propagation and the tunable optical response of graphene, a molecular sensor based on this newly proposed topological metasurface is designed and its sensitivity and functionality are quantitatively characterized.

Further author information: (Send correspondence to Nicolae C. Panoiu)

Nicolae C. Panoiu: E-mail: n.panoiu@ucl.ac.uk 


\section{TOPOLOGICAL VALLEY PLASMON TRANSPORT IN GRAPHENE BI-LAYER METASURFACES}

In this section a graphene bi-layer metasurface structure is designed to possess valley topological plasmonic modes in a nontrivial bandgap. The method used is to break the spatial-inversion symmetry of the graphene structure by horizontally shifting the two halves of the hole lattice of the top layer by a certain distance, $s$, as shown in Fig.1. As a consequence, the three-dimensional (3D) band structure shows a nontrival gap which is opened at the symmetry-protected Dirac point. Under these circumstances, topological edge modes are observed along a mirror symmetric interface generated by combining together two graphene bi-layer metasurfaces. In addition, we show that the light propagation along the mirror-symmetric interface of the topological edge modes displays unidirectional propagation features.

\subsection{Optical Properties of Graphene}

Graphene is a 2D-material platform that can be use to achieve topologically protected plasmonic modes, due to its extremely high carrier mobility and long life-time. ${ }^{9-11}$ Recently, advances in nanofabrication techniques have made possible the fabrication of graphene structures suitable to study topological plasmonics. ${ }^{12,13}$ The optical properties of graphene are described by its electric permittivity $\epsilon_{g}$, which can be evaluated by Kubo' formula: ${ }^{14}$

$$
\epsilon_{g}=1-\frac{e^{2}}{4 \epsilon_{0} \pi \hbar \omega h_{g}} \ln \left(\frac{\xi-i \bar{\omega}}{\xi+i \bar{\omega}}\right)+\frac{i e^{2} k_{B} T \tau}{\epsilon_{0} \pi \hbar^{2} \omega \bar{\omega} h_{g}}\left[\frac{\mu_{c}}{k_{B} T}+2 \ln \left(e^{-\frac{\mu_{c}}{k_{B} T}}+1\right)\right]
$$

where $\omega$ is the frequency, $\bar{\omega}=1-i \omega \tau$, and $\xi=2 \tau\left|\mu_{c}\right| / \hbar$. In our simulations, $T=300 \mathrm{~K}, \tau=50 \mathrm{ps}, h_{g}=0.5 \mathrm{~nm}$ and $\mu_{c}=0.2 \mathrm{eV}$ are the temperature, relaxation time, graphene thickness, and chemical potential, respectively. Unless otherwise specified, the default value of the chemical potential of graphene is $0.2 \mathrm{eV}$.

\subsection{Modeling of Graphene Bi-layer Metasurfaces}

The schematic of the proposed graphene bi-layer metasurface is illustrated in Fig.1. The bi-layer metasurface is composed of two layers of graphene with the same rhombic unit cell. The two halves of the hole lattice of top layer are horizontally shifted in opposite directions w.r.t. the bottom lattice by a certain distance, $s$ after one of the two top lattices has been rotated by $\pi / 3$. As indicated in Fig. 1(a), this leads to the formation of a mirror-symmetric domain-wall interface along $x$-axis. As we will demonstrate in what follows, this interface behaves as a plasmonic waveguide that possesses topological plasmonic modes. The hole lattices of the top and bottom layers consist of hexagonal arrays of holes with radius, $r$, the lattice constant being $a$. The top view and bird's eye view of the unit cell are given in Fig.1(b) and 1(c), respectively. Here, we use a $a=400 \mathrm{~nm}$, the hole radius $r=100 \mathrm{~nm}$, and the separation distance between the top and bottom layers, $h=90 \mathrm{~nm}$.

\section{(a)}

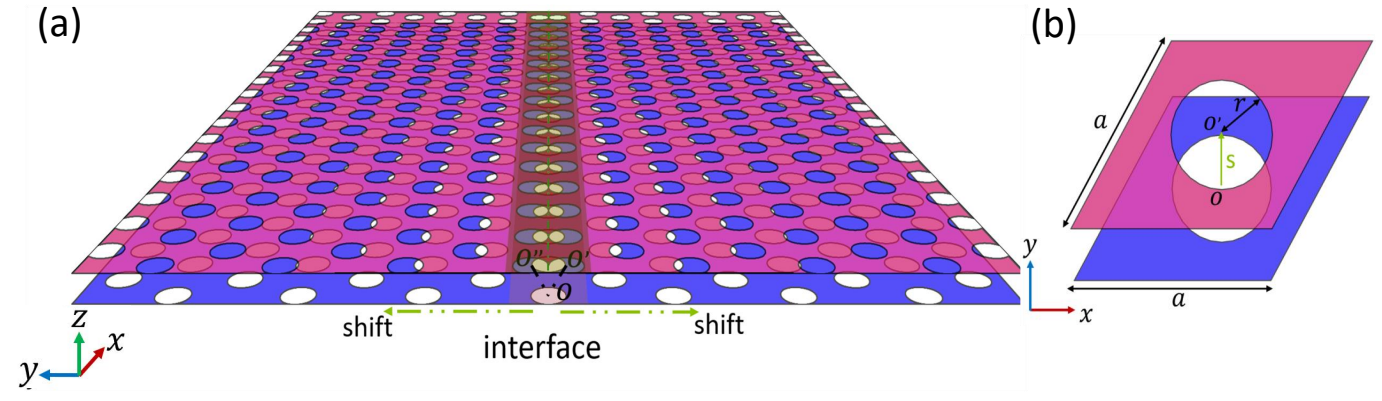

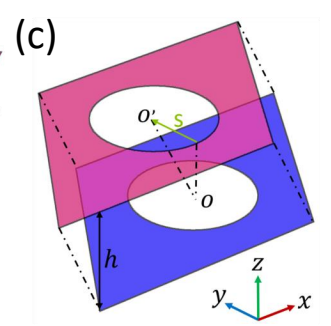

Figure 1. Schematic of graphene bi-layer metasurfaces. (a) Bi-layer metasurfaces is composed by shifting two halves of top graphene layer (fuchsia) with respect to the bottom graphene layer (purple) along $+y$ and $-y$ axis, which generates a domain-wall interface with a mirror symmetry. (b) Top view of the unit cell with a shift distance $s$ along y-axis between the top garphene later with respect to the bottom graphene layer. $O$ and $O^{\prime}$ correspond to the centers of the bottom and top rhombic cells with lattice constant $a$, respectively. (c) Three-dimensional view of the unit cell with a separated distance $h$ of two layers along z-axis. 


\subsection{Band Structure Analysis}

Unlike a hexagonal lattice of holes, which has the symmetry points called $\Gamma, K$ and $M$, the positions of high symmetric points in the first Brillouin zone (FBZ) of the 3D lattice, as depicted in Fig. 1(c), are no longer the same as those in the $2 \mathrm{D}$ case. ${ }^{15}$ Thus, the band diagram of this $3 \mathrm{D}$ graphene metasurface in the whole FBZ has been determined, the results being presented in Fig. 2. When the distance $h$ between the top and bottom graphene metasurfaces is large, namely the optical coupling between them can be neglected, each graphene metasurface possesses decoupled Dirac cones. ${ }^{16}$ Figure 2(a) indicates the overlap of Dirac cones of the two graphene layers. The frequency of the six degenerate Dirac points is about $14 \mathrm{THz}$. In order to enhance the coupling between the top and bottom graphene metasurfaces, the separation distance $h$ is reduced to $h=90 \mathrm{~nm}$, which results in a strong near-field coupling. Figure 2(b) shows the effect of this interaction. Thus, due to the strong coupling between the metasurfaces, it possible to achieve a symmetry-inversion breaking by a introducing a horizontal shift, as described above. In turn, this symmetry breaking perturbation leads to the formation of a topological nontrivial bandgap at the location of the Dirac points, which extends from $13.96 \mathrm{THz}$ to $14.17 \mathrm{THz}$.

Starting from these ideas, we determined the band diagram of a properly designed graphene bi-layer metasurface and the results are given in Fig. 3(a). The topologically protected edge modes are marked by red lines while the dark red regions represent the projection of bulk modes. As a structure with finite width along $y$-axis (10 unit cells), the graphene bi-layer metasurface embedded air has other types of edge modes, too, marked with blue lines in Fig.3(a). These modes are formed at the edges of the metasurface and are trivial modes. The edge modes that are locates at these edges generally appear in pairs inside the gap, e.g. points (1) and (2) and points (3) and (4) shown in Fig.3(a).

In order to gain deeper physical insights into the physics of these two different types of edge modes, we determined the field distributions of the modes corresponding to the points marked in Fig. 3(a). Thus, Fig. 3(b) and Fig. 3(c) show the field profiles of the edge modes located at the upper and lower boundary of the metasurface, respectively. The points (1) and (2) and points (3) and (4) in Fig. 3(a) indicate two pairs of edge modes located at the opposite boundaries of the metasurface, and can be clearly distinguished from topological interfacial modes. Moreover, the first pair of edge modes correspond to pure guided modes whereas the latter

(a)

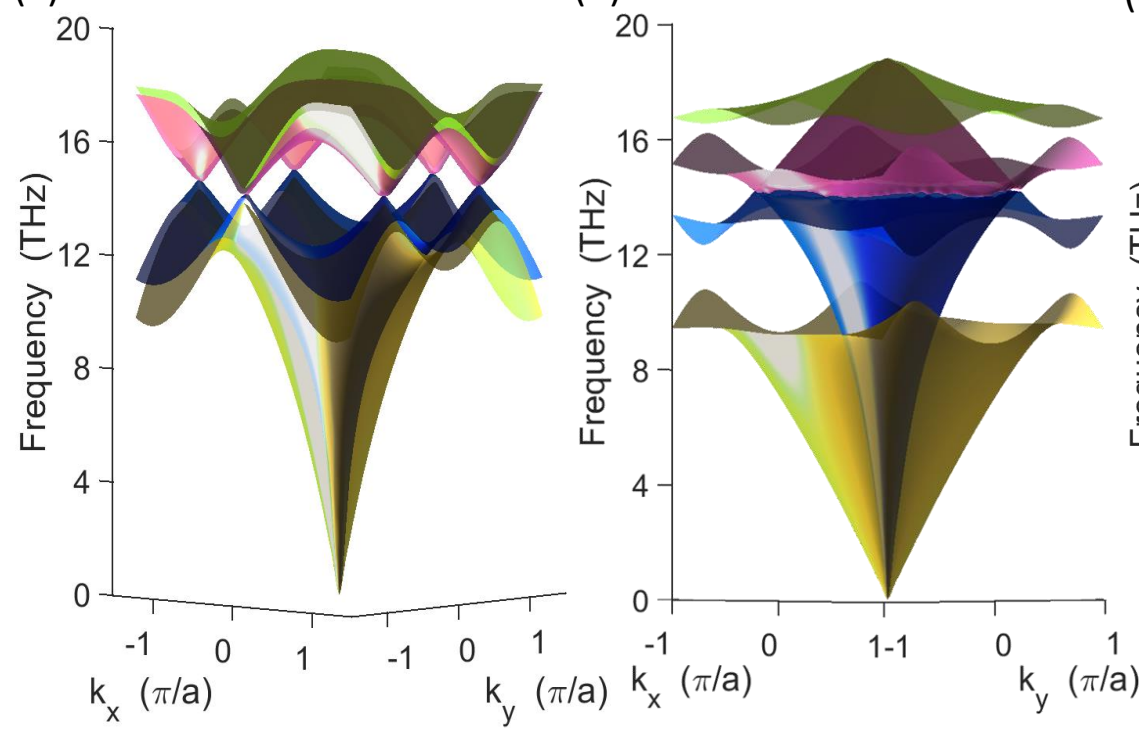

(c)

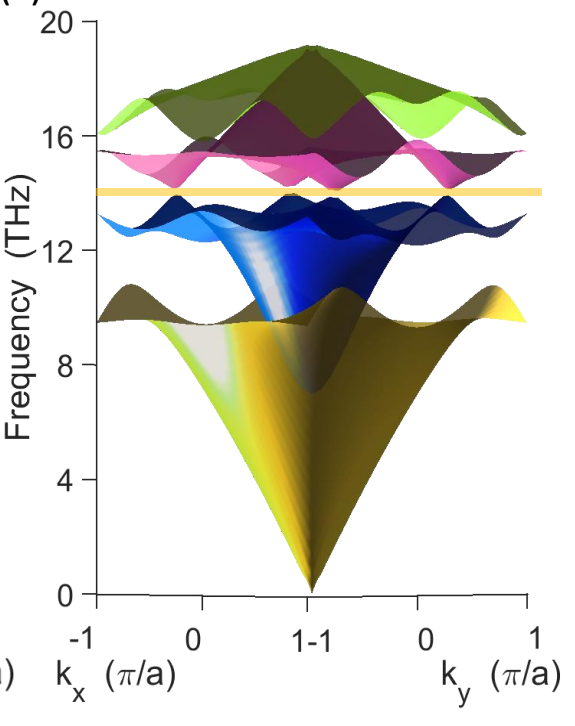

Figure 2. 3D band diagrams of bilayer graphene metasrufaces in the FBZ. (a) Band diagram when the weak coupling between the top and bottom graphene metasurfaces could be neglected. (b) Band diagram when the coupling between the top and bottom graphene metasurfaces is enhanced by reducing the separated distance $h=90 \mathrm{~nm}$. (c) Band diagram in which a $100 \mathrm{~nm}$ shift distance $s$ between the top and bottom graphene metasurfaces causes the spatial-inversion symmetry broken, where the distance $h=90 \mathrm{~nm}$ and the shift $s=100 \mathrm{~nm}$ is demonstrated in Fig. 1(c), a nontrivial gap emerges in the frequency range from $13.96 \mathrm{THz}$ to $14.17 \mathrm{THz}$. 

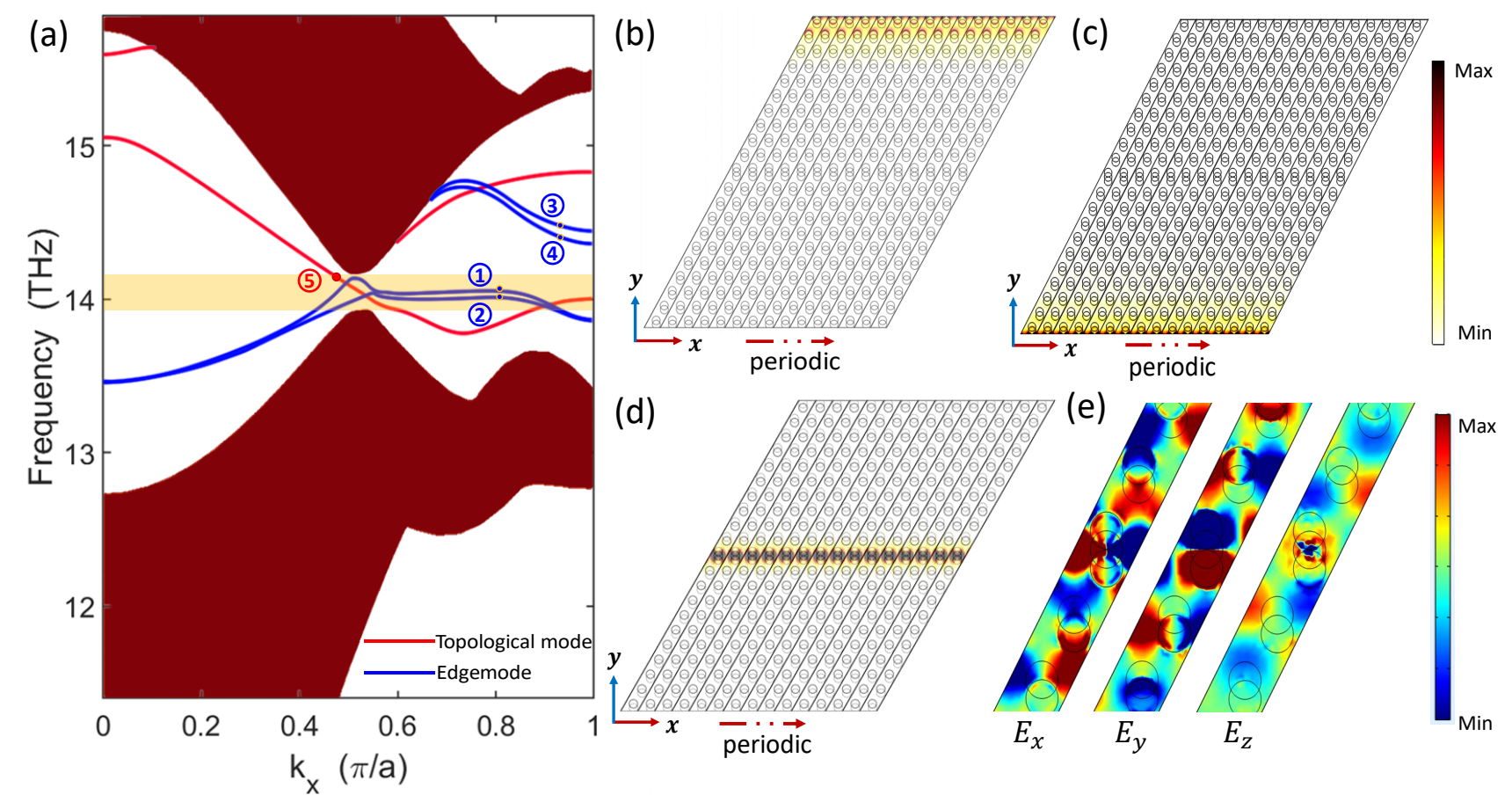

(e)

Figure 3. (a) Band diagram of a finite bilayer graphene metasurface with a width of 10 unit cells and domain-wall interface generated by shifting two halves of the top layer w.r.t the bottom graphene layer $(s=100 \mathrm{~nm})$. Topological interface modes and non-topological edge modes are represented by red and blue lines, respectively, whereas the bulk modes correspond to the maroon region. (b) Field distribution of a non-topological edge mode located at the upper boundary of the graphene metasurfaces, marked by (1) and (3) in (a). (c) Field distribution of a non-topological edge mode located at the lower boundary, marked by (2) and (4) in (a). (d) Field distribution of a topological interface mode marked by point (5) in (a). (e) Spatial distribution of the $E_{x}, E_{y}$, and $E_{z}$ components of the topological modes marked by red lines in (a).

one to leaky guided resonances. Furthermore, at the frequency of $14.16 \mathrm{THz}$, located inside the bandgap, there is another mode indicated in Fig. 3(a) by the red dot (5), the corresponding field distribution being presented in Fig. 3(d). This field profile clearly shows that the optical field of this mode is confined at the domain-wall interface. The symmetry properties of the interfacial topological modes are illustrated in Fig. 3(e), where we present the spatial profiles of the three components of the electric field. Thus, with respect to the interface, the field components $E_{x}$ and $E_{z}$ are symmetric whereas the field component $E_{y}$ is antisymmetric.

\subsection{Topological Properties of Interfacial Modes}

Our computational investigations demonstrate an important property of the interfacial topological modes, namely their unidirectional propagation. Thus, in practice, the topological graphene metasurface is generally a finite finite system, namely the graphene metasurface is truncated in $x$ - and $y$-direction. In order to break the periodicity along the $x$-axis, absorption boundary conditions are used for the $x$ and $y$-axis, whereas free boundary conditions are used for the $z$-axis. In Fig. 4(a), the line corresponding to the frequency $14.16 \mathrm{THz}$ is inside the bandgap and only crosses the topological bands at two points, i.e. points (1) and (2). These two points have opposite $k$ vectors and sign of the slope of their corresponding dispersion curves, which leads to mode propagation in opposite directions along the domain-wall interface.

In order to study the valley-Hall chirality-locking property of the topological interfacial modes, a source of monochromatic circularly polarized light with frequency $14.16 \mathrm{THz}$ is placed at the domain-wall interface. More specifically, six electric dipoles with phase that increases clockwise are placed at the six corners of a hexagonal unit cell, with the phase difference between adjacent dipoles being set to $\pm \pi / 3$, so as to generate left-circularly polarized (LCP) waves and right circularly polarized (RCP) waves, respectively. In the case of RCP excitation, as shown in Fig.4(b), the topologically protected mode only propagates along the interface in a negative $x$-axis 

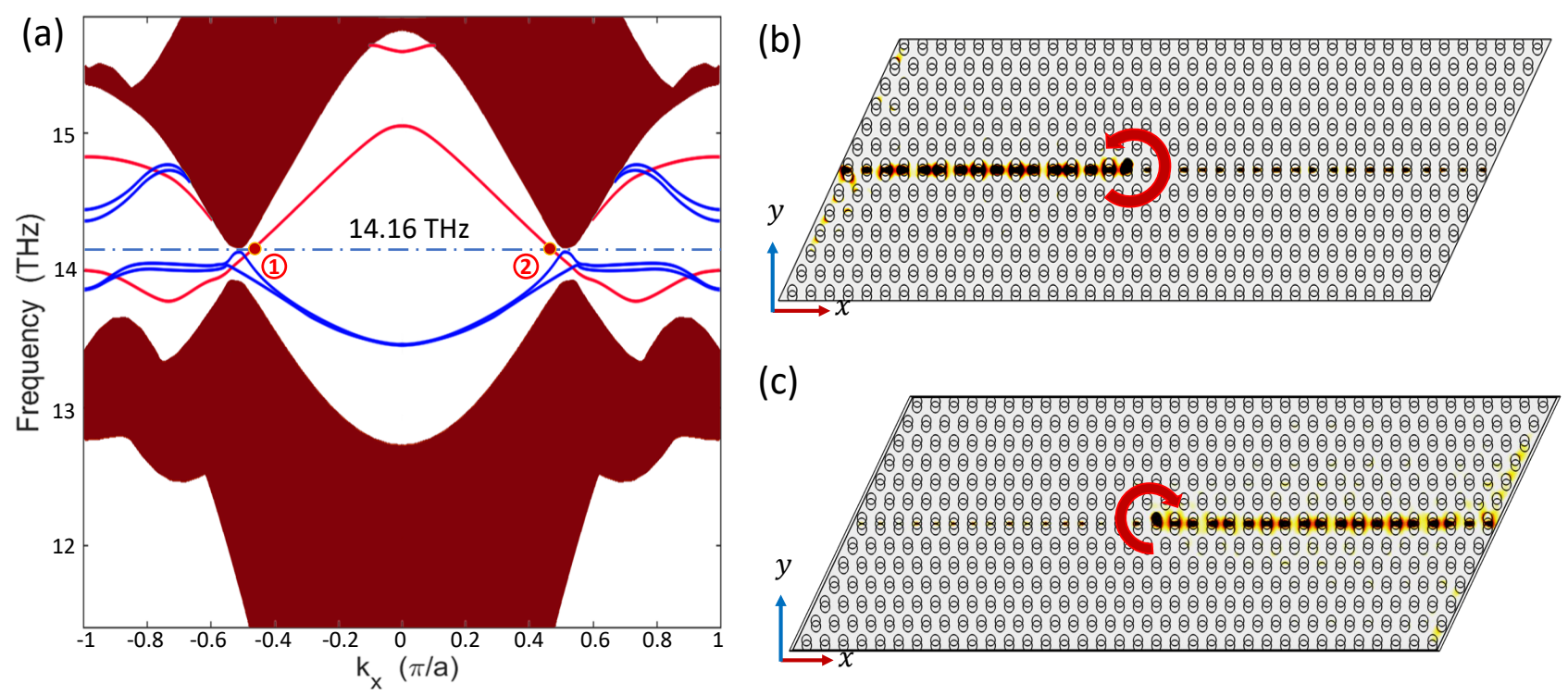

(c)

Figure 4. Unidirectional propagation of topological interface modes along the domain-wall interface at $14.16 \mathrm{THz}$. (a) Projected band diagram in which there are two clear topological edge states (points (1) and (2)) with opposite slopes at the frequency $14.16 \mathrm{THz}$. (b) Unidirectional propagation along $-x$-axis, marked by (1) in Fig.4(a), when the finite graphene metasurfaces is excited by a right circularly polarized (RCP) source. (c) Unidirectional propagation along $+x$-axis, marked by (2) in Fig.4(a), when the finite graphene metasurfaces is excited by a left circularly polarized (LCP) source.

direction, which corresponds to point (1) in Fig. 4(a). By contrast, the mode propagates in the positive $x$-axis direction in the case of LCP excitation, corresponding to the point (2) in Fig. 4(a).

\section{GRAPHENE-BASED GAS MOLECULAR SENSOR}

The unidirectional propagation feature of the topological interface mode of the bilayer graphene metasurface investigated in this work can find applications to efficient photonic nanodevices. To illustrate this, in what follows we demonstrate how the interfacial topological mode can be used to design a molecular sensor. Thus, graphene is a particularly promising $2 \mathrm{D}$ material for sensing applications, chiefly due to its tunable chemical potential and high optical damage threshold.

Taking advantage of the unidirectional propagation feature of the topological interface mode in the proposed bilayer graphene metasurfaces, an efficient molecular sensor has been designed. Graphene is an ideal 2D-material platform for sensing, chiefly because of its highly tunable chemical potential and large optical damage threshold. The chemical potential $\mu_{c}$, is a key physical parameter of graphene, that is proportional to the Fermi velocity and the carrier density, $n_{0}$. The chemical potential of graphene could be tuned via the molecular doping as a well-known type of chemical doping, ${ }^{17}$ which can be used to engineer the chemical potential of graphene with high precision. By detecting the concentration of adsorbed gas molecules, this graphene-based sensor could measure the gas concentration in the environment. The schematic of the proposed graphene-based molecular sensor is illustrated in Fig. 5(a). The sensor consists of three graphene bi-layer metasurface regions, namely region I, II and III. The length of each region is $l_{1}, l_{2}$, and $l_{3}$, respectively, and the initial chemical potential of each region is $0.2 \mathrm{eV}$, namely $\mu_{c 1}=\mu_{c 2}=\mu_{c 3}=0.2 \mathrm{eV}$. The input LCP source is placed at the center of the region I, and the output power is collected in the region III. The region II is exposed to the environment and detects the gas molecules.

The induced concentration of electrons or holes is increased due to the gas molecules adsorbed in the region II, which results in the increment of the graphene chemical potential $\mu_{c 2}$ in the region II. The variation of garphene permittivity leads to a shift of the corresponding frequency of the topological nontrivial bandgap of region II. When the frequency of the input light in region I is fixed inside the band gap at $14.16 \mathrm{THz}$, the light propagation 
(a)

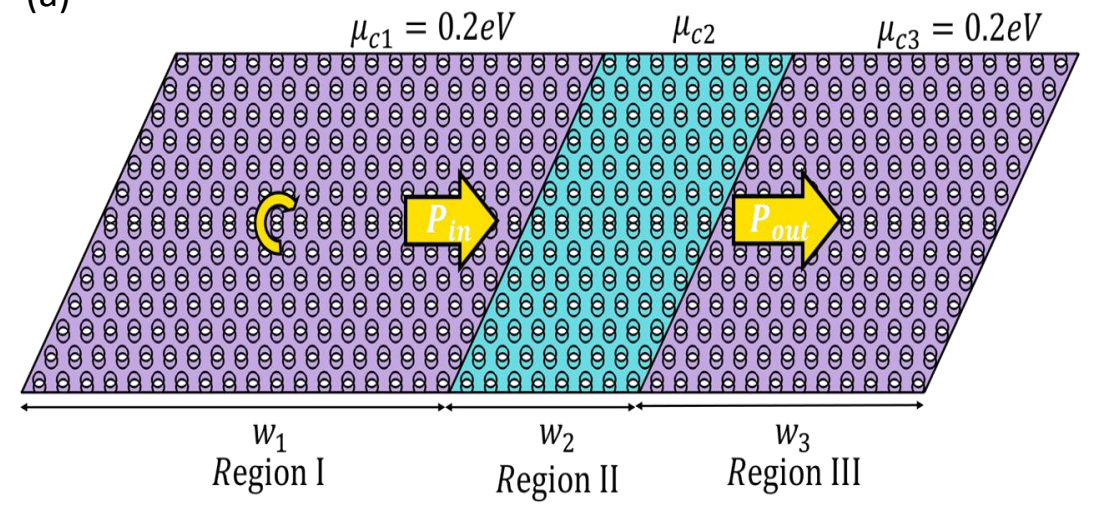

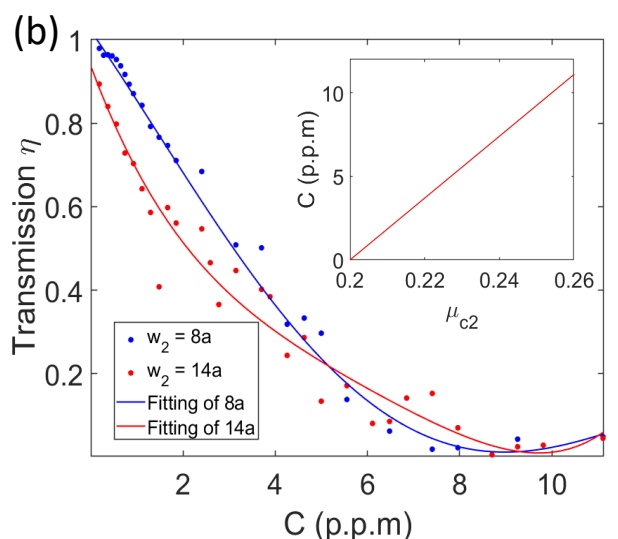

Figure 5. Molecular sensor based on bilayer graphene metasurfaces. (a) Schematic of the graphene-based molecular sensor. The input LCP source is pumped at the center of region I, the output power is measured in region III. The middle region II is designed to detect the concentration of the $\mathrm{NO}_{2}$ gas molecules, based on the fact the chemical potential of this region would be changed if there are gas molecules adsorbed on it. (b) Transmission of the topological mode with respect to the concentration $C$ of $\mathrm{NO}_{2}$ gas molecules in the presence of $w_{2}=8 a$ and $w_{2}=14 a$. The inset shows the linear relation between the $\mathrm{NO}_{2}$ concentration $\left(\mathrm{C}_{\mathrm{NO}_{2}}\right)$ and the corresponding chemical potential $\mu_{c 2}$ in region II.

mode in region II can be switched from a guided interfacial mode into a leaky bulk mode. Since the topological mode propagating from the region I is scattered and lossy in the region II, the output power $P_{\text {out }}$ measured in region III will rapidly decease.

In order to verify this prediction, and quantify the performance of the proposed sensor, we have studied the transmission of the topological mode through the three-region structure. To be more specific, in many experiments experiments ${ }^{18-20}$ the harmful gas $\mathrm{NO}_{2}$ is studied, so we consider this gas in our analysis, too. As shown in the upper inset of Fig. 5(b), the linear relation between chemical potential change $\Delta \mu_{c 2}$ and the concentration of $\mathrm{NO}_{2}$ gas molecules is expressed as $\Delta \mu_{c 2}=\alpha C_{\mathrm{NO}_{2}}$, where the linear parameter $\alpha \approx 5.4 \times 10^{-3}$ $\mathrm{eV} /$ p.p.m is taken from measurements. ${ }^{20} \mathrm{~A}$ monochromatic input power of $0.2 \mathrm{~W}$, at the frequency $14.16 \mathrm{THz}$, is inserted at the center of the region I. The length of region I $\left(l_{1}\right)$ and region III $\left(l_{3}\right)$ are $18 a$ and $12 a$, respectively. Using full-wave simulations, we calculated the transmission of the molecular sensor, which is defined by the ratio between the output power $P_{\text {out }}$ measured in region III and the input power $P_{\text {in }}$ in region I, namely $\eta=P_{\text {out }} / P_{\text {in }}$. In two cases corresponding to two different lengths of region II $\left(l_{2}=8 a\right.$ and $\left.l_{2}=14 a\right)$, as shown in Fig. 5(b), a large variation of transmission $\eta$ with respect to the gas concentration $C_{\mathrm{NO}_{2}}$ is observed. The dots indicate the simulated results, and are fitted by a three-order polynomial function marked by the solid lines. As expected, the longer the length $w_{2}$ of the region II is, the larger the slope of the transmission curve is. When the concentration $\mathrm{C}_{\mathrm{NO}_{2}}$ is increased to 9.26 p.p.m, most of the input power of topological interfacial modes is radiated and scattered, which means that the transmitted power and the light transmission both approach to zero. Moreover, the sensitivity of this graphene-based molecular sensor is defined as the absolute value of the first-order derivative of transmission with respect to the concentration of gas molecules detected in region II, namely $\sigma=\left|\eta^{\prime}\left(C_{\mathrm{NO}_{2}}\right)\right|$. Our simulation results show that the corresponding average sensitivity of this graphene-based molecular sensor can be as large as 0.2 . When the harmful gas molecules are adsorbed onto the metasurface, the variation of transmission $\eta$ of the power can indicate the concentration of gas molecules. This phenomenon can lead to a novel gas molecular graphene sensing application with a high sensitivity.

\section{CONCLUSION}

In conclusion, we presented a graphene bi-layer metasurface structure that possesses valley-Hall topological plasmonic modes. By creating a horizontal shift of the top graphene metasurface with respect to the bottom one, the spatial-inversion symmetry of the graphene plasmonic crystal is broken so that a nontrivial valley bandgap with a bandwidth of $0.21 \mathrm{THz}$ is formed. When a mirror symmetric domain-wall interface is constructed, topologically protected edge modes emerge inside the topologically nontrivial bandgap and the corresponding light propagation shows unidirectional features. We also demonstrated that by tuning the chemical potential of 
graphene via chemical adsorption of gas molecules, one can shift the frequency of the corresponding bandgap. This phenomenon has been subsequently used to design a sensitive molecular sensor. This work may open up a new way to achieve novel plasmonic sensors ${ }^{21}$ and other efficient photonic devices.

\section{ACKNOWLEDGMENTS}

This work has been supported by the European Research Council (ERC), Grant Agreement no. ERC-2014-CoG648328, China Scholarship Council (CSC) and University College London (UCL).

\section{REFERENCES}

[1] Khanikaev, A. B. and Shvets, G., "Two-dimensional topological photonics," Nat. Photonics 11, 763 (2017).

[2] Lu, L., Joannopoulos, J. D., and Soljacic, "Topological photonics," Nat. Photonics 8, 821 (2014).

[3] Ozawa, T., Price, H. M., Amo, A., Goldman, N., Hafezi, M., Lu, L., Rechtsman, M. C., Schuster, D., Simon, J., Zilberberg, O., and Carusotto, I., "Topological photonics," Rev. Mod. Phys. 91, 015006 (2019).

[4] Siroki, G., Huidobro, P. A., and Giannini, V., "Topological photonics: From crystals to particles," Phy. Rev. $B$ 96, 041408 (2017).

[5] You, J. W., Lan, Z., and Panoiu, N. C., "Four-wave mixing of topological edge plasmons in graphene metasurfaces," Sci. Adv. 6, eaaz3910 (2020).

[6] Ju, L., Shi, Z., Nair, N., Lv, Y., Jin, C., Velasco Jr, J., Ojeda-Aristizabal, C., Bechtel, H. A., Martin, M. C., Zettl, A., et al., "Topological valley transport at bilayer graphene domain walls," Nature 520, 650 (2015).

[7] Lan, Z., Celi, A., Lu, W., Ohberg, P., and Lewenstein, M., "Tunable multiple layered Dirac cones in optical lattices," Phys. Rev. Lett. 107, 253001 (2011)

[8] Tse, W., Qiao, Z., Yao, Y., MacDonald, A. H., and Niu, Q., "Quantum anomalous Hall effect in single-layer and bilayer graphene," Phy. Rev. B 83, 155447 (2011).

[9] Jin, D., Christensen, T., Soljacic, M., Fang, N. X., Lu, L., and Zhang, X., "Infrared topological plasmons in graphene" Phys. Rev. Lett. 118, 245301 (2017).

[10] You, J. W., Lan, Z., Bao, Q. and Panoiu, N. C., "Valley-Hall topological plasmons in a graphene nanohole plasmonic crystal waveguide," IEEE J. Sel. Top. Quantum Electron. (submitted) (2020).

[11] You, J., Bongu, S., Bao, Q., and Panoiu, N. C., "Nonlinear optical properties and applications of 2D materials: theoretical and experimental aspects," Nanophotonics 8, 63 (2018).

[12] Zhang, Y., Zhang, L., and Zhou, C., "Review of chemical vapor deposition of graphene and related applications," Acc. Chem. Res. 46, 2329 (2013).

[13] Norman, J. J. and Desai, T. A., "Methods for fabrication of nanoscale topography for tissue engineering scaffolds," Ann. Biomed. Eng.. 34,89 (2006).

[14] Goncalves, P. A. D. and Peres, N. M., An introduction to graphene plasmonics, (World Scientific, 2016).

[15] Kittel, C., McEuen, P., and McEuen, P., Introduction to solid state physics, Vol. 8 (Wiley New York, 1996).

[16] Xie, B., Wang, H., Zhu, X., Lu, M., Wang, Z., and Chen, Y., "Photonics meets topology", Opt. Express 26, 24531 (2018).

[17] Rodrigo, D., Limaj, O., Janner, D., Etezadi, D., De Abajo, F. J. G., Pruneri, V., and Altug, H., "Midinfrared plasmonic biosensing with graphene", Science 349, 165 (2015).

[18] Schedin, F., Geim, A. K., Morozov, S. V., Hill, E., Blake, P., Katsnelson, M., and Novoselov, K. S., "Detection of individual gas molecules adsorbed on graphene", Nat. Mater. 6, 652 (2007).

[19] Hu, H., Yang, X., Guo, X., Khaliji, K., Biswas, S. R., de Abajo, F. J. G., Low, T., Sun, Z., and Dai, Q., "Gas identification with graphene plasmons", Nat. Commun. 10, 1131 (2019).

[20] Novoselov, K. S., Geim, A. K., Morozov, S., Jiang, D., Katsnelson, M. I., Grigorieva, I., Dubonos, S., Firsov, and A. A., "Two-dimensional gas of massless Dirac fermions in graphene", Nature 438, 197 (2005).

[21] Anker, J. N., Hall, W. P., Lyandres, O., Shah, N. C., Zhao, J., and Van Duyne, R. P., "Biosensing with plasmonic nanosensors", in Nanoscience and Technology: A Collection of Reviews from Nature Journals 308, World Scientific, 2010. 